\title{
Spatial Diversity Deconstruct of C-band Scatter Components in Multistatic RaDaR Datasets using Machine Learning Techniques
}

\author{
Shanmugha Sundaram G A, Harun Surej I, Karthic S, Gandhiraj R, Binoy B N, Pradeep Kumar K A, and
}

Thiruvengadathan $\mathrm{R}$

\begin{abstract}
In complex application wherein the signal propagating through free space is subject to multipath interference due to scatter by line-of-sight and non-line-of-sight objects in the propagation channel. The aims is to identify scatter centers in the propagation channel and characterize them based on their subjective characteristics, interpreted based on machine learning algorithm operations. Data-driven models are employed, replacing the traditional analytical approaches, in order to profile the scatter centers as either of absorbing or reflecting types based on the manner in which the signals are affected. A typical multistatic detection scenario is reconstructed under controlled laboratory conditions in order to create spatially independent data sets, while operating in the $C$-band frequency. The outcomes of this study are then applied to identify the scatter centers based on the distinct signatures they register in the experimental data set. As a converse argument, the process of antenna pattern estimation can now be performed free of an anechoic chamber setup, which is time and cost insensitive. A greater relevance shall be in the context of mid-band 5G-NR cellular communication systems that need to optimize the distributed antenna location attributes on time and cost constrained scales before attempting a large-scale deployment.
\end{abstract}

Index Terms-Signal processing antennas, Microwave radio communication, Multipath channels, RaDaR detection, Radio propagation terrain factors, Scattering.

\section{INTRODUCTION}

Electromagnetic waves are used in a wide range of critical applications in an increasingly globalized world. In situations that range from pear-to-pear transmission all the way up to interstellar satellite communications, the primary objective has been to transfer information through a propagating channel [1], [2]. A propagation channel has an unpredictable response to radio waves traveling across it, as they incorporate undesirable factors such as distortion and scattering in the form of multipath propagation contributed by line of sight (LoS) and non-LoS objects [3],[4]. These scatterers in the propagation channel irregularly diffuse, reflect or refract light in many directions as defined by their physical properties which leads to severe deterioration of the signal quality. As a result, the signal is received with a significant amount of additive noise by the receiver antenna. To enable effective and reliable communication, it is important to characterize the channel both quantitatively and qualitatively.

The defining of a robust model for the scatterers has been a major challenge. Typically, they are modeled using analytical formulations, or by conducting channel measurement campaigns. These approaches are powerful as they rely on a deep understanding of the system and can benefit from scientifically established relationships [1]-[4]. An instance of this is the impact of the marine environment to wireless communication that was studied by placing a transmitting antenna on a moving ship and a receiver antenna on land [1]. C-band signals, with a bandwidth of $100 \mathrm{MHz}$, were employed in the experiments, and measurements were taken to study the scattering effect over the sea. Apart from the direct LoS transmission between the transmitter and receiver, the interaction between the signal and the sea surface was studied. This interaction was studied using the Karasawa's model [2], that defines signal parameters such as amplitude and phase. The results of this seminal work have proven that Karasawa's model still holds good for the specific choice of carrier frequency and propagation environment.

The scattering effects in underwater propagation medium were studied in [4]. The frequency of transmission ranged all the way from $2-32 \mathrm{kHz}$ that revealed a wide diversity of propagation effects and scattering conditions. Jie Zhou et al. [5] used a generalized three-dimensional (3-D) scattering model that considered the transmitter to be placed at the center of a 3-D spheroidshaped region, and the scatterers are assumed to be uniformly distributed within the radius of this region. The work led to developing a model that performs significantly better when compared to previously developed 2-D models.

The work is supported in the form of equipment provided by National Instruments Corporation, TX, USA under its Academic Research Grant of 2016; the front line RF resources and equipment had been integrated and supported in the form of onsite maintenance by the NI Systems India Pvt. Ltd., KA, India; these are gratefully acknowledged by the authors.

All authors are with the Department of Electronics and Communication Engineering, Amrita School of Engineering, Coimbatore, Amrita Vishwa Vidyapeetham, India (Phone: 0091-422-2685000; e-mail: ga_ssundaram@cb.amrita.edu).

All authors are also affiliated with the SIERS Research Laboratory, Amrita School of Engineering, Coimbatore, Amrita Vishwa Vidyapeetham, India 
A geometrical model was developed by Lu et al [6] in which a $6 \cdot 10^{4} \times 6 \cdot 10^{4} \mathrm{~m}^{2}$ box had been centered is the setup within the $10^{4} \times 10^{4} \mathrm{~m}^{2}$ test region. The antennae were placed randomly within the box. The received power was then computed and stored to analyze the results. Similar simulations are carried out for the available different artificial terrain databases. Various modeldriven approaches have been proposed in the past for both indoor and outdoor environments in order to characterize the propagation channel. The uniform ring model proposed in [7] was the first geometry-based channel model. A novel 2-D geometry-based stochastic channel model has been proposed in [8] as an extension of the unified disk scattering model. A detailed geometric scattering model for a vehicle-to-vehicle communication channel is discussed by Cheng et al [9], where the scatterers were assumed to be uniformly distributed along the path of the communication channel.

Given the inherent empirical nature associated with such schemes, finding a suitable model and refining it until it produces the desired results is often a tedious process [10]. Due to recent advancements in computation, it is now possible to model the propagation channels using an entirely data-driven approach relying heavily on several potent machine learning (ML) algorithms. The work reported here aims to characterize the propagation channel In order to create a comprehensive profile of as many of the scatterers in a given signal space. The data processed using the proposed methodology had been collected in a controlled laboratory environment. The advantage of these algorithms over traditional model-driven approaches is that they are adaptive to change in environment and types of inputs, while enabling a quick estimation of the channel parameters.

In terms of the derived benefits to the antenna characterization process, a study of the sort reported here can pave the way for an eventual and comprehensive replacement of existing techniques that rely significantly on exorbitantly expensive facilities such as the anechoic chamber. With a thorough understanding of the scatter component in the received radio frequency (RF) signals, this data-driven method can serve as a desktop computer based antenna design and performance evaluation scheme, making the RF front-end fabrication exercise highly cost effective.

The frequency band considered for evaluation in this work is significant in the context of the fifth generation new radio (5GNR) wireless technology in digital cellular networks. Of the 2 bands allocated for this service, Frequency Range 1 (FR1) constitutes the mid-band $5 \mathrm{G}$ in the sub- $6 \mathrm{GHz}$ band, offering the furthest reach in terms of abilities to negotiate terrestrial obstacles than FR2 (the mm-wave band) [11]. The 3GPPs vision of pervasive 5G communication includes the usage of spacebased and airborne high-altitude platform station (HAPS) network nodes. A 5G network will have to incorporate extensive testing, that optimizes its performance in an eventual deployment, based on factors such as propagation analysis and coverage mapping. This shall also ensure a peak performance in the related systems against various other negative attributes such as RF interference, noise and distortions. Site characterization for eventual location of antennas, that are an elaborate exercise making use of extensive outdoor test range equipment, can be attempted convincingly with the approach suggested in this work, augmented with of space-based, signal coverage models driven by a need for enhanced situational awareness.

The paper is organized in the following manner: a study of the prior art is succeeded by an extensive discussion related to the experimental setup, hardware specifications, data acquisition and pre-processing stages. This is followed by the section that explains the systematic approaches adopted in data analysis using ML techniques, and salient interpretations of prominent the scatter signatures. The paper is concluded with a poignant note on the potential impact that this research work shall have in the domain of antenna and RF system design.

\section{METHODOLOGY}

\section{A. $\quad$ Experimental Setup}

The indoor floor map of the laboratory is depicted in Figure 1. It gives an overview of the layout of equipment vis-a-vis the diverse range of scatter centers that exist as furniture and civil infrastructure. This floor map has been used to validate the role of predominant scatter centers from results that analyze the experimental data obtained in a generic, representative physical design of the laboratory.

Omni-directional linear dipole antennas, as 3 deployed units of the type VERT2450 of Ettus Research LLC [12], with dual band frequency responses in the 2.4-2.48 GHz and 4.9-5.9 GHz range and gain of $3 \mathrm{dBi}$, were used in this experimental setup. One antenna was assigned to the transmit (Tx) side, and the other two antennas were with the receive (Rx) channels. One of the receivers (RX-1) was placed in direct LoS with the transmitter antenna and the other one (RX-2) was placed at variable angle $\theta$ with respect to the bore-sight alignment. This was to detect any possible spatial variations due to the presence of preferential scatterers located at different angles with respect to the receiver antenna. This off-boresight angle was changed from $15^{\circ}$ to $60^{\circ}$ in increments of $15^{\circ}$. 


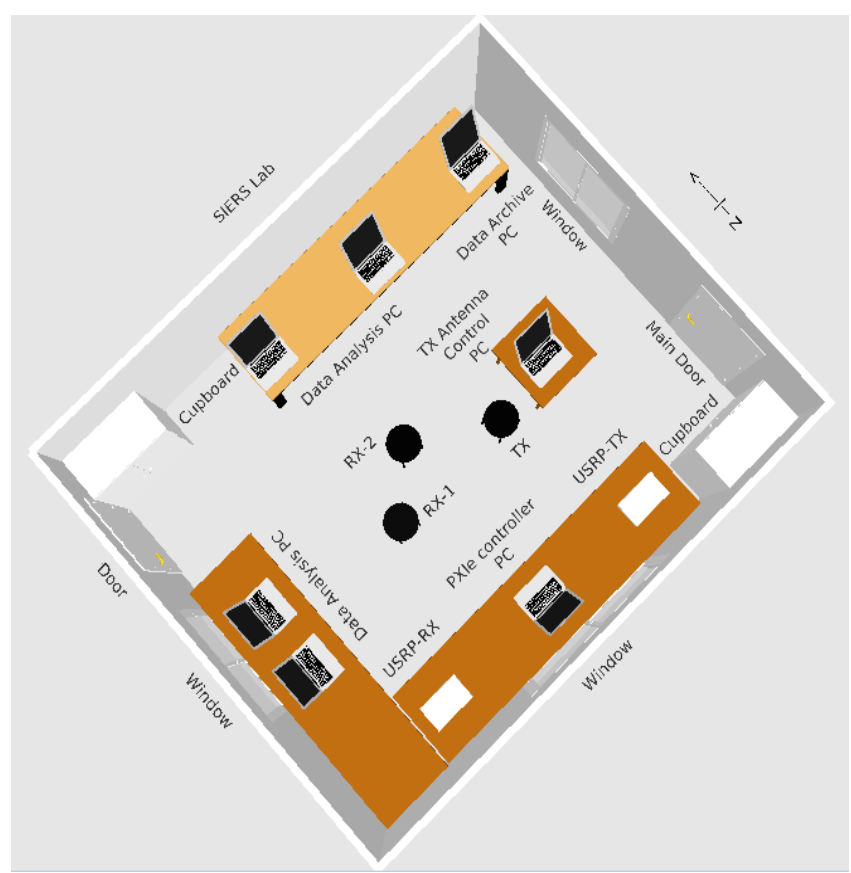

Fig. 1. Indoor floor map of the SIERS Research Laboratory.

The Tx-Rx arrangements in the experiment are shown in Figure 2. Two units of the GPS-disciplined universal software radio peripheral (USRP), each with upto $4 \mathrm{Tx}-\mathrm{Rx}$ channels, $160 \mathrm{MHz}$ instantaneous bandwidth, and an operating frequency range 10 MHz to $6 \mathrm{GHz}$ [13] had been configured in a manner such that, one serves as the Tx (single channel) and one as the Rx (dual receive channels). The overall system is controlled using a PXIe controller [14] that operates in a LabView [15] environment. The Tx-Rx system design had been integrated as a software defined radio using the Communications System Design (CSD) suite [16] that generates a single tone $100 \mathrm{kHz}$ sine signal which was transmitted at a bandwidth of $400 \mathrm{kHz}$ in the C-band frequency $(5.25 \mathrm{GHz})$.

\section{TX}

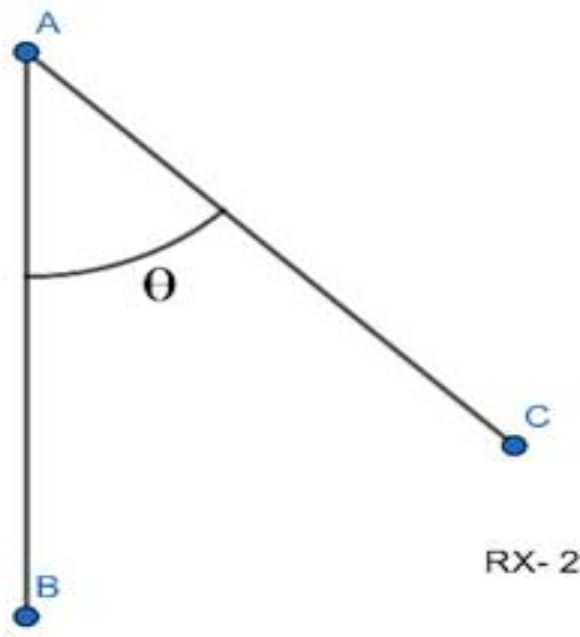

$R \times-1$

Fig. 2. Experimental setup of the transmitter antenna (TX) and the Receiver antennas (RX -1 and $R X-2)$. 
In order to obtain all round coverage of the transmit antenna radiation pattern that corresponds to data over all $360^{\circ}$, the transmitter antenna was made to rotate about a vertical axis, using a dedicated Arduino-driven [17] stepper motor, controlled by a Raspberry Pi-2 (Model B) [18] single board computer (R-Pi SBC).

Spatial data at enhanced granularity by spatially sampling across an angular span of $0.698^{\circ}$, resulting in 516 discrete positions over the $360^{\circ}$ in the elevation (E) plane. At each angle, eight snapshots of the data were collected. Each of these snapshots comprised 20000 data points. In a similar manner, five independent voltage-time series data sets for various off-boresight angles were collected and used for analysis.

For a transmitter resistance $\mathrm{R}=50 \Omega$, and a root mean squared (RMS) value of the transmitter voltage $\left(\mathrm{V}_{\mathrm{rms}}\right)$, the transmitted power is then given by [19]:

$$
V_{r m s}=V^{2} / 2=0.25 V
$$

where the transmitter voltage $\mathrm{V}$ is $0.707(\mathrm{~V})$. The transmitter power is then given by [12]:

$$
P_{g}=V_{r m s} / R=5 \mathrm{~mW}
$$

Here, the radiation resistance on the transmit side is $\mathrm{R}=50 \Omega$. The power radiated by the transmitter antenna would then be:

$$
P_{t}=g_{t} / g_{t|\max |} \times P_{g}=315.6 \mu \mathrm{W}
$$

where $g_{\mathrm{t}}=20 \mathrm{~dB}$ is the gain of transmitting side amplifier, and the maximum gain $g_{\mathrm{t}(\max )}$ with which the USRP transmits is $32 \mathrm{~dB}$, when there is a maximum radiated power of $100 \mathrm{~mW}$.

\section{B. $\quad$ System-wide simulation}

The process of deconstructing a C-band real time $\mathrm{RaDaR}$ system starts with simulations that employ codes developed using the LabView CSD Suite. These software codes are deployed to generate the time series raw data representing an antenna's radiation pattern. The codes implement transmission of a sinusoidal message tone at $100 \mathrm{kHz}$ modulated using a $5.25 \mathrm{GHz}$ carrier into free space and retrieves the message tone through two receiver antennas separated spatially.

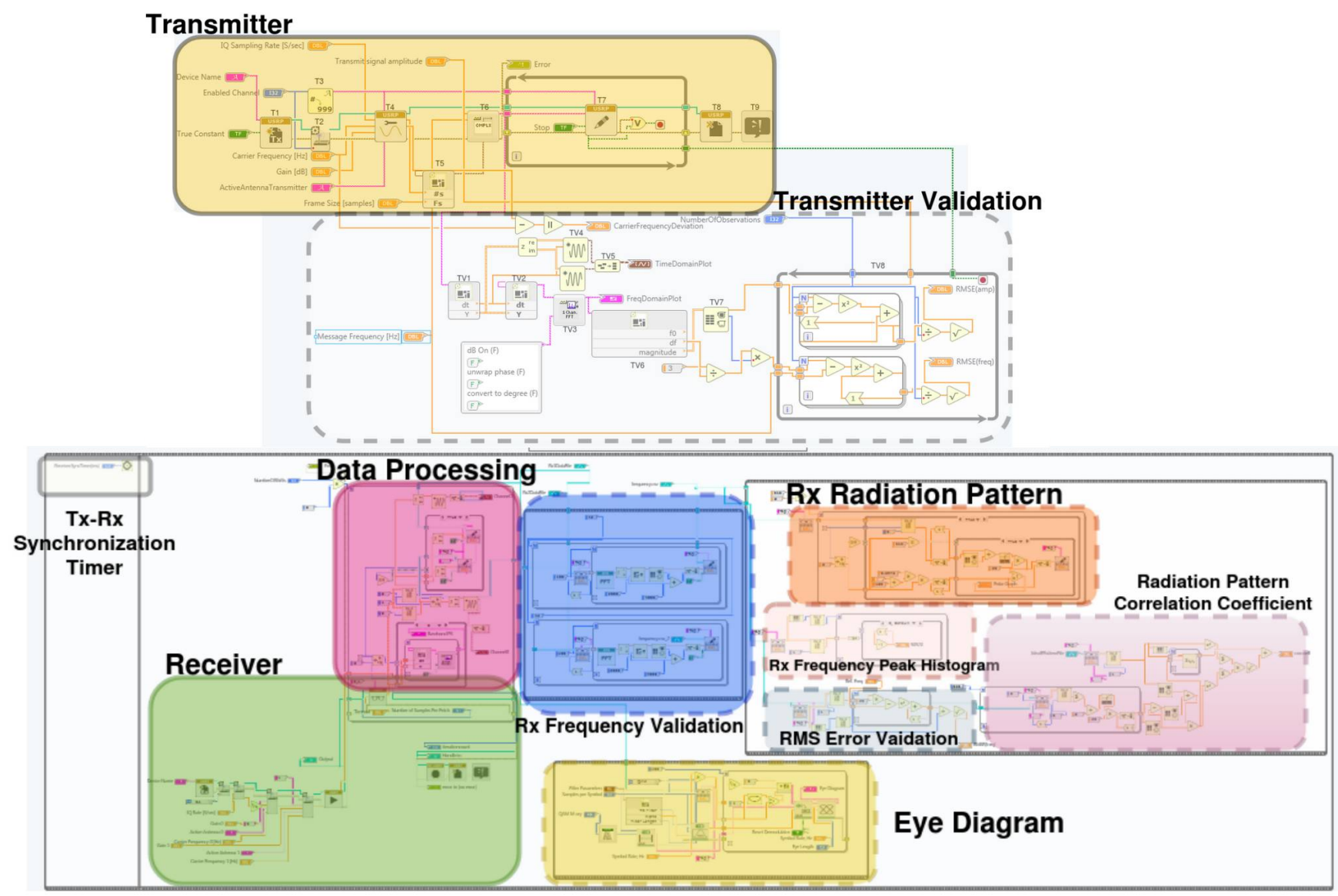

Fig. 3. LabVIEW virtual instrument designed for the radiation pattern. 
The LabVIEW implementation diagram shown in Figure 3 is the graphical representation of the source codes that are salient parts in the RaDaR system deconstruct exercise. The LabVIEW software codes primarily enable a control of the USRP transmitter and the receiver in order to achieve a robust transmission and the reception process. The data acquisition system shown here consists of two subsystems: Transmitter, and the Receiver-cum-Data processor subsystems. Besides the primary blocks, the various other functional blocks indicated in the figure support the primary blocks in deploying various validation metrics such as Rx frequency validation, Rx bit error rate (BER) validation (derive vital inferences from the eye diagram), and radiation pattern correlation coefficient. A visual validation is also obtained from a radiation pattern plot and a receive frequency histogram.

TABLE I

SOFTWARE CONFIGURATION PARAMETERS

\begin{tabular}{ll}
\hline \multicolumn{1}{c}{ Parameter } & \multicolumn{1}{c}{ Value } \\
\hline Device ID (DI) & RIO1 \\
Carrier signal frequency (CSF) & $5.25 \mathrm{GHz}$ \\
Message signal frequency (MSF) & $100 \mathrm{kHz}$ \\
Sampling/IQ rate (SR) & $400 \mathrm{ksps}$ \\
Transmitter gain (TG) & $10 \mathrm{~dB}$ \\
Receiver gain (RG) & $10 \mathrm{~dB}$ \\
Transmit signal amplitude (SA) & $1-\mathrm{V}$ sinusoidal peak-peak \\
Active Antenna of Transmitter & TX1 \\
(AAT) & \\
Enabled channel transmitter (ECT) & 0 \\
Active Antenna of Receiver (AAR) & RX1 \\
Enabled channel receiver (ECR) & 0,1 \\
Number of samples retrieved (NSR) & $800 \mathrm{k}$ \\
& \\
\hline
\end{tabular}

The software configuration parameters are listed in Table I. The carrier signal frequency corresponds to the resonant frequency of the transmit and receiver antenna. The USRP hardware defines a minimum sampling rate of 390k samples per second (ksps), and a more practical sampling rate of $400 \mathrm{ksps}$ had been considered during the data acquisition phase.

The sampling rate has to be kept as low as possible to minimize hard-disk storage consumption. As per the Nyquist criterion, the theoretical maximum message signal frequency that can be reliably detected using a sampling rate of $400 \mathrm{ksps}$ cannot exceed half the sampling rate, i.e., $200 \mathrm{kHz}$. The Nyquist rate being the theoretical minimum sampling rate, in practice, the sampling is done at a much higher rate to improve frequency resolution and signal-to-noise ratio [19]. Oversampling by a factor of 2 has been applied here, and this has resulted in a message signal frequency that is equivalent to a fourth of the sampling rate, i.e., 100 $\mathrm{kHz}$. The USRP hardware allows the transmit and the receive gain varied from $0 \mathrm{~dB}$ to $31.5 \mathrm{~dB}$ corresponding to a transmit power that ranges from $50-100 \mathrm{~mW}$. The transmit and receive gain parameters are chosen as $10 \mathrm{~dB}$ after a careful observation had been made during the process of signal acquisition.

The scheme to data acquisition involves a coordinated activity among the various modules that are a part of the RaDaR simulation system, depicted as a workflow diagram in Figure 4. The program control waits on an infinite loop till the user activates the start transmission control button. On receiving the start transmission signal, the USRP transmitter is configured using the configuration parameters - DI, CSF, SR, TG, SA, AAT, and ECT — as mentioned in Table I.

Time series data samples representing a sinusoidal voltage message signal of constant amplitude SA, frequency MSF are created and written to the data port of the USRP transmitter. The remaining processes needed for transmission, such as digital to analog conversion and modulation, are implemented internally by the USRP hardware, for which explicit coding is not required. Eventually, the modulated signal is transmitted using the antenna connected to the transmit port.

On the receiver side, the program control waits on an infinite loop until the user activates the start reception button. On this cue, two ports in the USRP receiver is configured using DI, CSF, SR, RG, AAR, and ECR (as listed in Table I). A receiver antenna is connected to each of the data ports. The demodulation of the received signal is carried out to retrieve the message signal internally by the USRP hardware, for which again explicit coding is not necessary. The samples representing the voltage of the recovered message signal is made available to the program control.

The program reads $800 \mathrm{k}$ (NSR) samples from each of the two data ports of the USRP receiver. From the samples that have been read, 8 segments, each of $100 \mathrm{k}$ samples, are created. The first $20 \mathrm{k}$ samples of each slice are stored in a single row of a spreadsheet, with each sample value occupying a single cell of the spreadsheet. The remaining 80k samples are discarded to minimize hard-disk storage consumption. In this manner, the signal is obtained in multiple time-slots rather than in a single slot, 
which aids to combat burst errors [20]. The experiment requires $10 \mathrm{~GB}$ of storage size without discarding the data, which is now reduced to $2 \mathrm{~GB}$. Storing 20k samples allows for a frequency resolution as low as $400 \mathrm{k} / 20 \mathrm{k}=20 \mathrm{~Hz}$ [21]. The read and store process consumes 2 seconds, referred to as the dwell time. Dwell time refers to the duration of halt in the transmit antenna orientation at a specific angle. After the fixed dwell time lapses, the transmit antenna is rotated through $0.697^{\circ}$ about a fixed vertical axis to the next angular position.

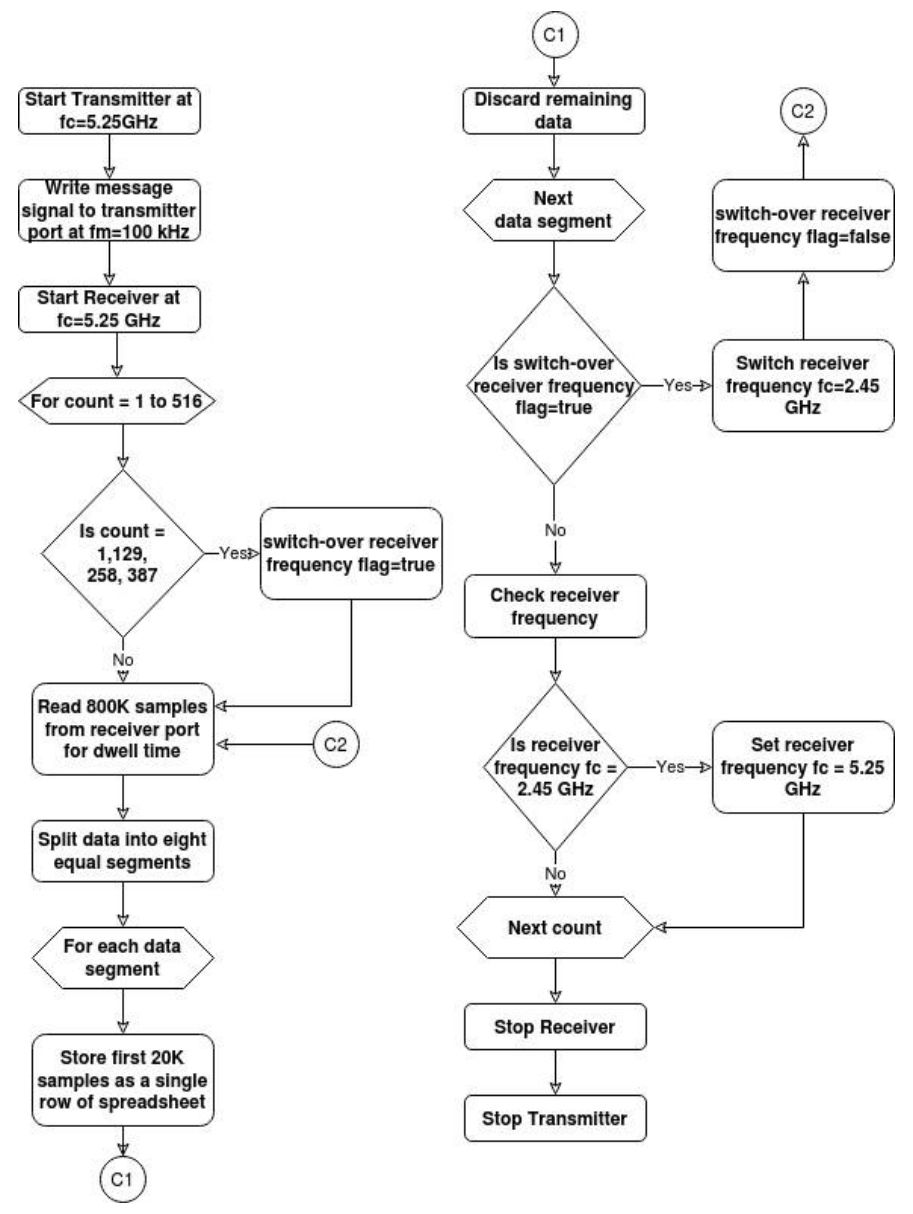

Fig. 4. Algorithm depicting the process flow for the radiation pattern time series data creation.

Steering of the transmit antenna is automated, and has been performed asynchronously using a stepper motor, controlled using an Arduino board and the R-Pi SBC. Though it is desirable to decrease the dwell time, it was observed that lowering the dwell time below 2 seconds impacts the quality of radiation patterns inferred from the signal-to-noise ratio (SNR) while processing of the raw data. The reading and store process are repeated for the remaining angles until the transmit antenna rotates through $360^{\circ}$. In addition to the above process, at angles of $0^{\circ}, 90^{\circ}, 180^{\circ}$, and $270^{\circ}$, the receiver is briefly switched to $f_{\mathrm{c}}=2.45 \mathrm{GHz}$ for performing a frequency diversity analysis. The receiver frequency is set to $f_{\mathrm{c}}=5.45 \mathrm{GHz}$ once the received signal is recorded.

\section{C. $\quad$ Porting the Data}

In this research work, MATLAB R2019a had been used for data processing. The five independent data sets were employed for the analysis. Each of the five data sets is of size 1 GB. Each dataset contains 4096 rows and 20000 columns. The MATLAB function 'datastore' [22] was used to import the data into the processing environment, without the need to occupy the system memory on the desktop computer which has a 64-bit Xeon 8-Core CPU and 16 GB RAM running on a Windows 7 operating system.

\section{Data Pre-processing}

The power spectrum for the collected voltage time series data were obtained and the received power was found by extracting the magnitude of power at the frequency of the message signal. 


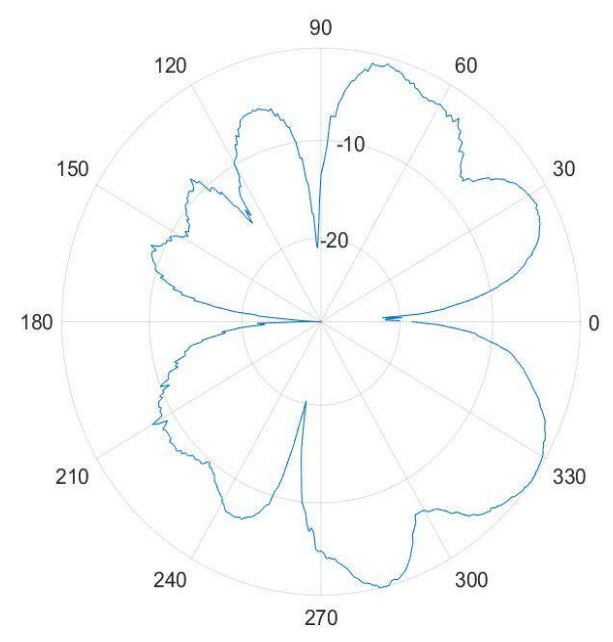

Fig. 5. Antenna radiation patterns in the elevation plane considered as the reference.

For the reference data regarding antenna radiation patterns, the manufacturer's data sheet [12] was consulted. To match the nulls of all the possible spatial configurations with this reference data set, shown for the elevation plane in Figure 5, the angular position at which there was minimum detected power was identified [23]. All the subsequent points were shifted such that they now coincide with this minimum power transfer angle, as depicted for the case of a $60^{\circ}$ off-boresight radiation pattern in Figures 6 ( $\mathrm{a}$ and $\mathrm{b}$ ), with $0^{\circ}$ being assigned as the reference boresight alignment axis. This angle-shifted data was then used for all further analyses.

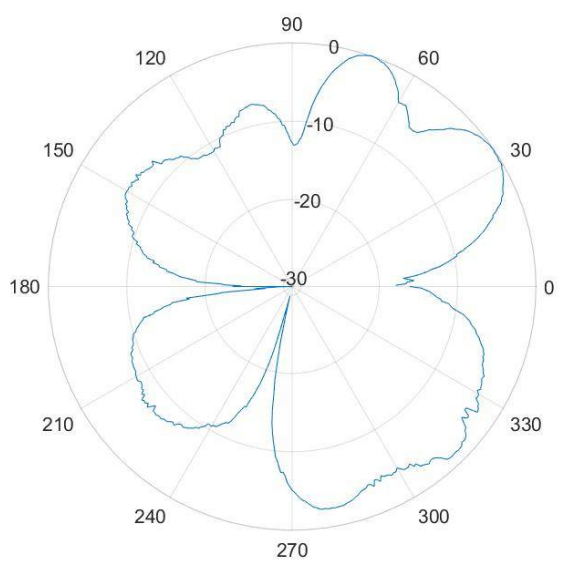

(a)

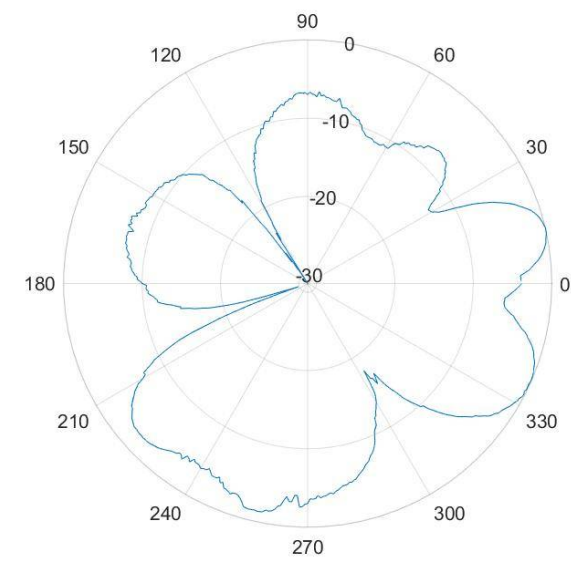

(b)

Fig. 6. Antenna radiation patterns: (a) before; and (b) after boresight alignment, for $\theta=60^{\circ}$.

The theoretical value of received power is obtained from the Friis radio link equation [22]:

$$
P_{r(t h)}=P_{t} G_{r} G_{t}\left(\frac{\lambda}{4 \pi r}\right)^{2}=0.26 \mu \mathrm{W}
$$

Here, $P_{\mathrm{t}}$ is obtained from equation (2), $G_{\mathrm{r}}=10 \mathrm{~dB}$ is the gain of the receiver antenna, $G_{\mathrm{t}}=1 \mathrm{~dB}$ is the gain of the isotropic transmitter antenna, $\lambda=0.0571 \mathrm{~m}$ is the wavelength of the transmitted signal, and $r=0.50 \mathrm{~m}$ is the distance between the transmitter and receiver. The received power was then converted into the decibel scale to obtain $R_{\mathrm{i}(\exp )}$ [24], that effectively is a difference between the $P_{\mathrm{i}(\exp )}$ (experiment-based value for signal power determined from the power spectrum of the C-band signal), and $P_{\mathrm{r}(\mathrm{th})}$ - the theoretical value of the received power as defined by equation (3). 


\section{E. $\quad$ Finding Residuals}

Residuals are an indication of how varied the experimental data is with respect to that of the reference data. This variation is attributed primarily to the presence of scatterers in the propagation channel.

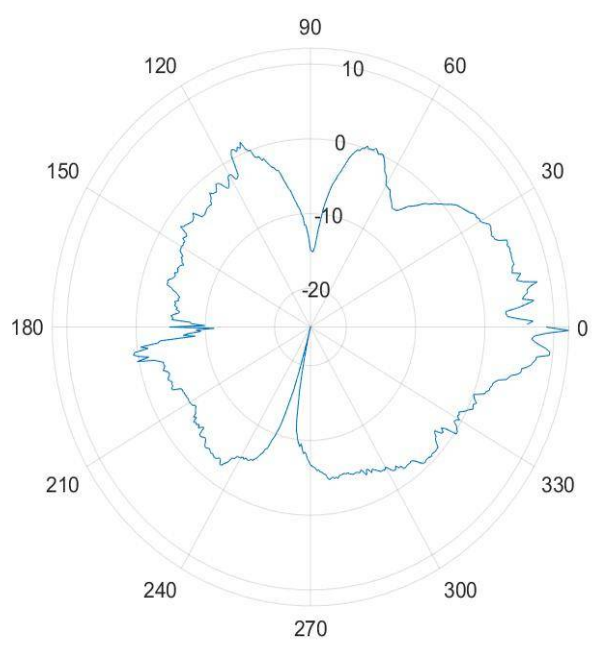

Fig. 7. Residual plot for $\theta=60^{\circ}$.

In order to detect and characterize the scatterers, residuals were determined and subjected to further analyses. The residuals were computed as:

$$
R_{i}=R_{i(\exp )}-D_{r e f}
$$

The first term on the right is the experimental received power value in $\mathrm{dBi}$, and the second one corresponds to the reference power value obtained from the antenna manufacturer's data sheet. In the further phases of analyses all such residuals were studied in terms of their angular distributions, in the form of polar plots similar to the one shown in Figure 7 for the case of a $60^{\circ}$ off-boresight orientation in RX-2. As a result, 5 additional residual plots in a polar reference frame were generated, that revealed the angular distributions in magnitudes of variation in the residuals.

\section{DATA ANALYSIS}

\section{A. $\quad$ Datasets}

The experimental data had been analyzed considering two different ways that had been identified:

i By taking the complete 4128 residual data per dataset by taking all 8 snapshots for each spatial position at once (516 spatial positions $\times 8$ snapshots per spatial position $=4128$ residual data points per dataset).

ii Residual data containing 516 points by taking one snapshot for all the spatial position (516 spatial position $\mathrm{x} 1$ snapshot $=516$ residual data points). There are 8 such snapshots per dataset. For the sake of simplicity, the $4^{\text {th }}$ snapshot of each dataset may be taken for analysis.

To prevent the loss of information from the experimental data, the $1^{\text {st }}$ approach was chosen to pursue the analysis.

\section{B. $\quad K$-Means clustering}

An exercise in classification of the scatter centers as either of absorbing or of reflecting types is based on their interaction with the C-band signals. A reflecting scatterer would leave an additive signature on the signal and an absorbing scatterer would create a diminishing effect on the signal. To classify and analyze the obtained residuals, K-means clustering is used, which classifies the residual data into ' $\mathrm{n}$ ' clusters.

K-means clustering had been chosen as the reference unsupervised learning method for data classification considering the diversity and size of the data being studied, and the eventual outcomes of analyses has a certain bearing on the particular context of the study being pursued [25]. Besides, this data-driven algorithm is also one of the simplest yet powerful when implemented for the dataset considered in the current work. The method aims at discovering inherent patterns in the data sets based on a measure of similarity [26]. The user has to define the number of clusters ' $n$ '. The initialization of the centroids is crucial, since 
the algorithm is highly sensitive to the choice of starting points [27], i.e., positioning of the initial centroids causes different clustering results.

One way to address this problem is to place the initial centroids such that the distances between them is the maximum. The aim is to minimize the sum of squared Euclidean distances between the points in the cluster to the centroid of that cluster. The squared Euclidean distances for an n-dimensional Euclidean space is defined as [28]:

$$
d^{2}(p, q)=\sum_{i=1}^{n}\left(p_{i}-q_{i}\right)^{2}
$$

where the point $p$ has the coordinates $\left(p_{1}, p_{2}, \ldots ., p_{n}\right)$ and the centroid $q$ has the coordinates $\left(q_{1}, q_{2}, \ldots ., q_{n}\right)$.

In order to extract all possible qualitative signatures of the potential scatterers from the dataset through clustering, it was necessary to determine the optimum number of clusters in the dataset. A very high probability of existence of more than 3 types of scatterers in the propagation channel was realized. For the current phase in the analyses, the value of ' $n$ ' was taken as 3 . To make the analysis even more effective, both power and spatial domain data were considered.

One shortcoming of this approach is that the centroids are initialized randomly for each value of ' $\mathrm{k}$ '. To determine the optimum number of clusters, iterative analysis using K-means clustering was done for different values of ' $k$ ', followed by a Silhouette analysis being performed on the output of K-Means clustering algorithm.

\section{Silhouette analysis}

Silhouette analysis is used as a measure to understand how an object in a cluster, is similar to the objects in its cluster, i.e. cohesiveness, in comparison with those in other clusters, i.e. separation [29]. The values of this silhouette analysis range anywhere from -1 to +1 , with -1 indicating worst possible clusters and +1 indicating best possible clusters. The silhouette score is calculated using the expression:

$$
S=\frac{1}{n} \sum_{i=1}^{n} \frac{\left(b_{i}-a_{i}\right)}{\max \left(a_{i}, b_{i}\right)}
$$

Given a cluster, $a_{i}$ is the mean of distances within the clusters and $b_{i}$ is the minimum nearest cluster distance, i.e., the distance to the point in the adjacent cluster. Clusters with an average silhouette value greater than 0.71 or higher are considered successful in determining the strong structures in input data [30].

\section{Iterative K-Means Clustering}

Silhouette analysis can also be used to determine the optimum number of clusters. This can be done by iterating the K-Means or $\mathrm{K}-$ Medoids clustering for a different number of clusters, ' $\mathrm{k}$ '. For instance, ' $\mathrm{k}$ ' can be varied from 1 to 30 . The variation of ' $\mathrm{k}$ ' with the average silhouette value can then be studied, and the value of ' $\mathrm{k}$ ' that results in the highest average silhouette value can be considered the most optimum clustering.

One issue observed in this approach is that the centroids are initialized randomly for each value of ' $\mathrm{k}$ '. The most optimum cluster is not considered for the computation of the silhouette value and the resulting average silhouette value is not the best possible value for that number of clusters. This means that even if clustering is repeated for the same value of ' $k$ ', say $n=5$, the resulting clusters are different each time. To address this issue, the method employed has been to iterate clustering multiple times for each value of ' $k$ ', say 20, and find the most optimum cluster by selecting the cluster that has the lowest Euclidean distance [6]. Each time the clustering process iterates, the centroids are randomized within the range of the dataset, ensuring that all of the possible combinations get explored. Thus, the algorithm ensures that the most optimum cluster for that particular value of ' $\mathrm{k}$ ' is always selected, and the average silhouette values are computed.

\section{E. $\quad$ K-Medoids clustering}

K-Means clustering algorithm is sensitive to outliers, i.e. the mean centroid is pulled towards the side that has the higher value of elements. Although the silhouette value obtained from the K-Means clustering [31] indicates good clustering, the centroids in the clusters are not within the dataset. To address this issue, K-Medoids clustering is used in place of K-Means clustering. KMedoids clustering uses the objects in the datasets as the centroids, instead of taking the mean value of the objects in the clusters as centroids [25]. This also ensures that the algorithm is immune to outliers and the centroid is located at the center of the dataset. So, K-Medoids clustering is used to cluster the data. Instead of assuming that the scatterers in the propagation channel would fall within the 3 categories of scatterers, i.e., absorbing, neutral or reflecting, it is better to conclude this from the available dataset. It shall then enable a robust decision to be made on the optimum number of clusters, and thereby identify the number of types of scatterers in the propagation channel. 


\section{F. $\quad$ Elbow Method}

The Elbow Method is one of the heuristic methods used to determine the optimum number of clusters. The within-cluster-sumof-squares (WCSS), 'J', is a measure of the sum of square of distances of each data point to their respective centroids. WCSS is defined by [32]:

$$
J=\sum_{i=1}^{k} \sum_{x \in C_{i}}\left|x-C_{i}\right|^{2}
$$

where ' $\mathrm{x}$ ' is the element of the cluster ' $\mathrm{C}_{\mathrm{i}}$ ' and ' $\mathrm{k}$ ' is the number of clusters $\left|\mathrm{C}_{\mathrm{i}}\right|$. As the number of clusters, ' $\mathrm{k}$ ', increases, WCSS, ' $\mathrm{J}$ ', decreases. When ' $\mathrm{k}$ ' is less than the optimum number of clusters, the decrease in WCSS is larger as an increase in ' $\mathrm{k}$ ' will greatly increase the degree of clustering of each cluster. When ' $\mathrm{k}$ ' is greater than the optimum number of clusters, the decrease in WCSS is diminutive as there is no good improvement in the degree of clustering. The WCSS characteristics would result in a graph which resembles that of an elbow. The value of ' $\mathrm{k}$ ' for which there is a reasonable decrease in the reduction of WCSS, after which the reduction in WCSS is minimal, is considered as the optimal number of clusters. The value of ' $\mathrm{k}$ ' at the 'elbow' is taken as the optimum number of clusters [33] when such an iterative analysis is completed.

\section{G. Correlation coefficient}

In order to understand the variability of clustering across the angular off-boresight datasets $\left(x_{\mathrm{i}}\right.$ for $\theta=15^{\circ}, 30^{\circ}, 45^{\circ}$ and $\left.60^{\circ}\right)$ correlate with the clusters in the bore-sight dataset $\left(y_{\mathrm{i}}\right.$ for $\theta=0^{\circ}$ ), the correlation coefficient had to be determined. The correlation coefficient, $\Gamma$, is a measure of the linear relationship between these pair of variables $\left(x_{\mathrm{i}}\right.$ and $\left.y_{\mathrm{i}}\right)$, and is defined by:

$$
\Gamma_{x y}=\frac{\sum\left(x_{i}-\bar{x}\right)\left(y_{i}-\bar{y}\right)}{\sqrt{\sum\left(x_{i}-\bar{x}\right)^{2} \sum\left(y_{i}-\bar{y}\right)^{2}}}
$$

The mean of the variable pair are denoted by $\bar{X}$ and $\bar{y}$, and $\Gamma$ values range from -1 to +1 . A value of 0 indicates no linear relationship; a value of +1 indicates a perfect positive linear relationship, while a value of -1 indicates a perfect negative linear relationship [34],

The correlation coefficient among the $\mathrm{k}$ clusters in each of the angular datasets (taken separately) vs. the k' clusters of the boresight dataset, would then result in a $(\mathrm{k} \times \mathrm{k}$ ') matrix. Each cell in the matrix corresponds to a correlation coefficient indicating the linear relationship between the two clusters taken; for e.g., the value in the fifth row, second column cell, corresponds to the correlation coefficient between the fifth cluster in the angular dataset and the second cluster in the boresight dataset.

To visualize the distribution in the correlation coefficients, a histogram plot has been generated. The bin size is decided to be 0.05 to enhance the resolution of the histogram. The correlation coefficient values that fall within that window are collected and the histogram is plotted for each dataset.

\section{H. Box plots}

A generic box plot, shown in Figure 8, is a standard way of representing data based on a 5-number summary [35]. This 5number summary consists of a minimum, the first quartile, the median, the third quartile and the maximum value of the given data set, and indicates the data distribution. The Min and Max in the box plot represent the minimum value and the maximum value of the data set, respectively. The first quartile (Q1) and the third quartile (Q3) represent the 25th and 75th percentiles of the data set, while the line marked in red is the median $(\mathrm{m})$ in the data set.

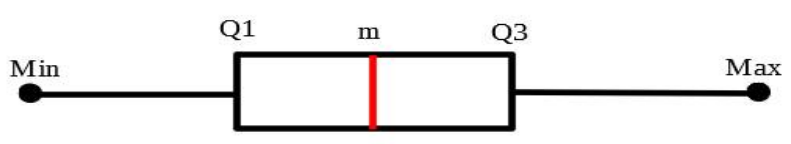

Fig. 8. Box plot and its five-number summary.

The size of the box is directly proportional to the diversity of the values; i.e., the closer the values, smaller the box and vice versa. To analyze the data quantitatively, two different box plots were generated. Every row in the experimental data was divided into 19 separate blocks, each consisting of 1024 data points; the power values were then determined from the power spectrum. Two different data processing approaches were followed:

(1) To understand the variance of power values across each angle, data which corresponds to the same angles were considered, and a box plot was generated. With each angle comprising 8 snapshots the combined yield is $19 \times 8=152$ power values for each angle. The resulting box plots contained 8 boxes, and each box is an indication of variation in these 19 values. 
(2) To understand the variance across all angles, a box plot was generated for all angles containing 516 boxes, where each box represents 19 values for that particular snapshot. A similar process was repeated for every snapshot, which yielded a total of 8 box plots.

\section{RESULTS AND DisCUSSION}

\section{A. Residual plot}

In this research work, K-Means clustering was done on the residual dataset to qualitatively differentiate the scatterers in the propagating channel. The number of clusters, ' $\mathrm{k}$ ', was set to 3 in the interest of classifying the scatterers as belonging to an absorbing, neutral or reflecting type. The clustering was done only in the power domain, ignoring the spatial domain, theta ' $\theta$ ', with the understanding that the signatures of scattering have a predominant presence in the power domain data. Small variations when compared with the ideal dataset are explained as contributed by the sensitive nature of the detection process that is able to sense the presence of randomized noise in the neighborhood of the experimental space - such as those due to the operation of undocumented radio equipment (mobile phones).

By choosing the number of clusters to be 3, the average silhouette value for the clusters is determined as 0.81544 , a clear indication of good clustering. The residuals analysis classifies the data set to be tightly grouped into three clusters. Since only the power domain was given as the input to the clustering algorithm, the algorithm had split the dataset into three partitions in the respective domain. Such distinct groupings are interpreted as qualitatively representing the scatterers in the propagation channel.

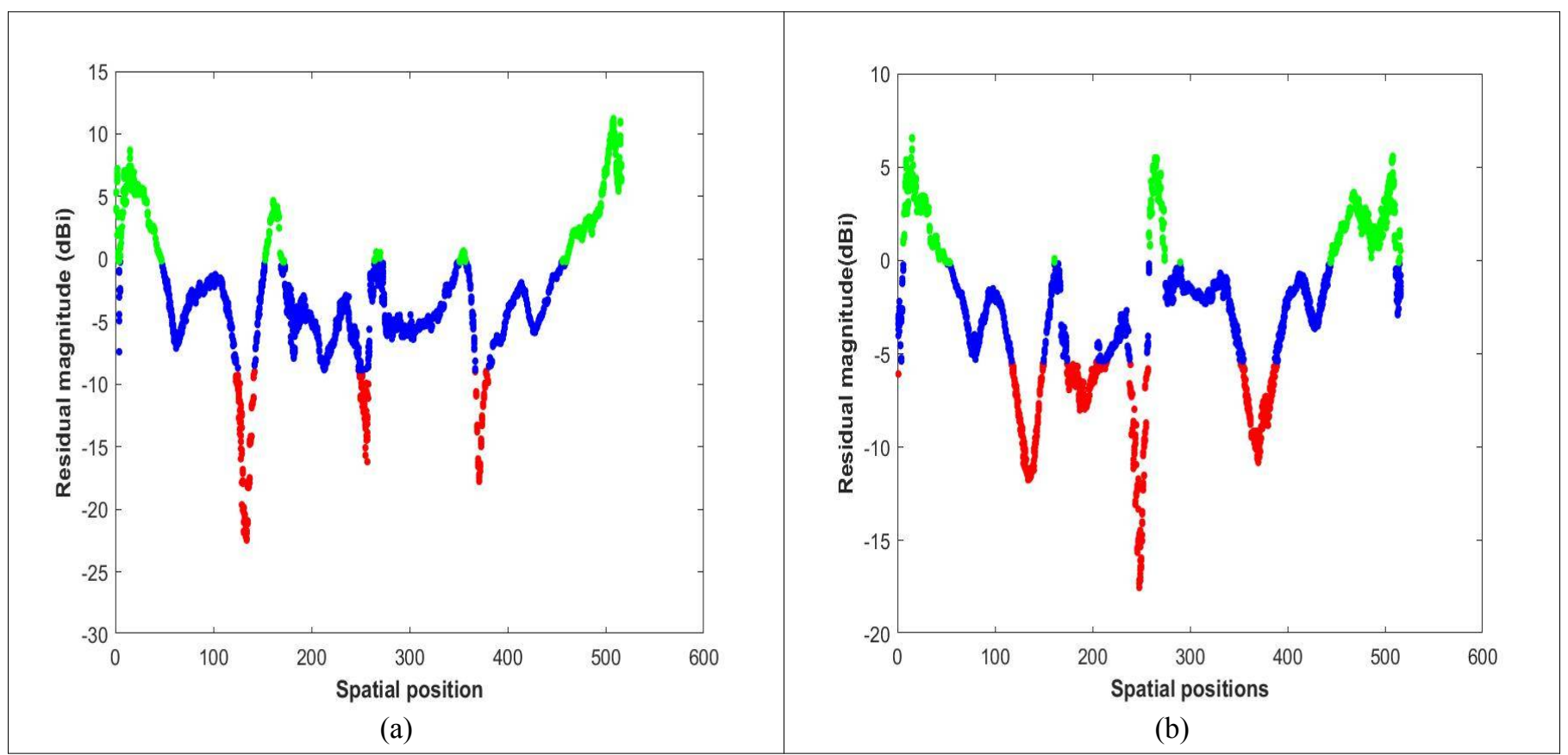

Fig. 9. Residuals grouped into 3 clusters for data from: (a) the LoS antenna (RX-1); and (b) the RX-2 antenna when placed at $\theta=15^{\circ}$.

Parameters such as the transmitter gain, receiver gain, transmitted power, distance of separation are all unknowns in the reference data set. So, a considerable variation from the reference set was expected and the results prove to be agreeable with this hypothesis. Groupings in the data set have also been noticed in cases corresponding to the four other off-boresight locations (such as the one shown in Figure 9 for $\theta=15^{\circ}$ ) that were also considered. The following observations have been made from the analyses:

1) The clusters represented by the color blue are distributed all along the data set. As is evident from the figures, data points in this cluster are distributed evenly and do not show extreme variations. Such a trend indicates that these residuals are not a definitive signature left by the scatterers in

the propagation channel, and their low-profile and ubiquitous presence is discounted in any further study.

2) The clusters represented by the color red are the set of data points in which the received power for signals are significantly low when compared to that of the reference data set. These set of points in the cluster arises due to the presence of absorbing scatterers which absorbed the incident signal and thus the power received by the receiver antenna is reduced. 
3) The clusters represented by the color green denote the spatial positions at which the received powers were relatively high compared to that of the reference data set. These set of points in the cluster arises due to the presence of reflecting scatterers which acts like a mirror and reflects the incident signal resulting in a higher power received by the receiver antenna.

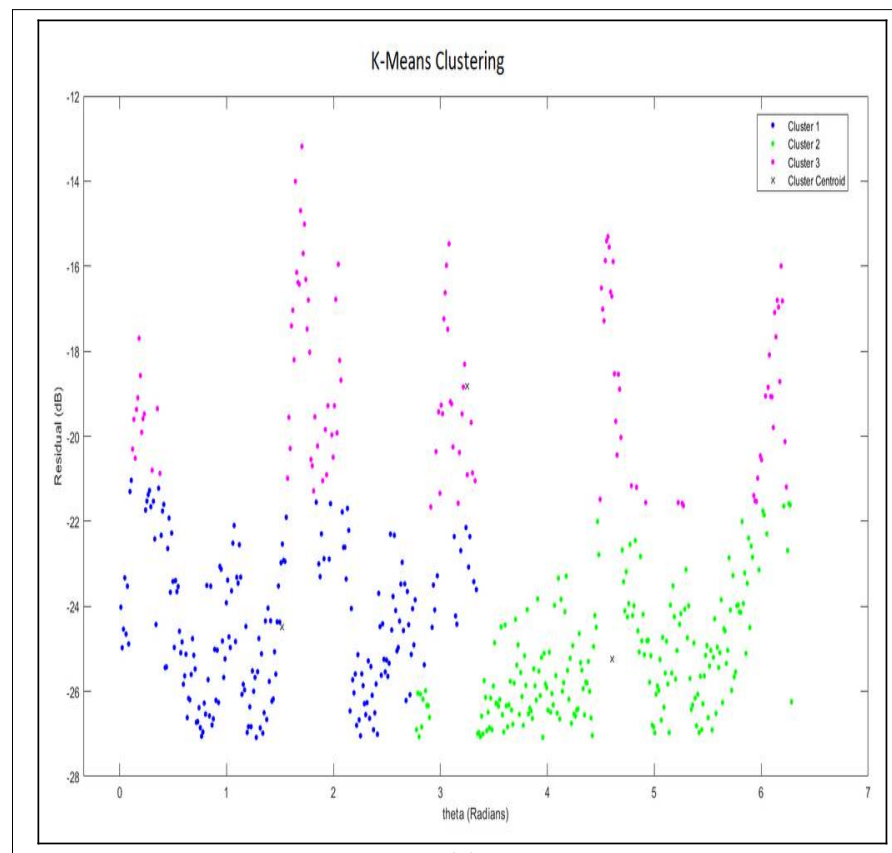

(a)

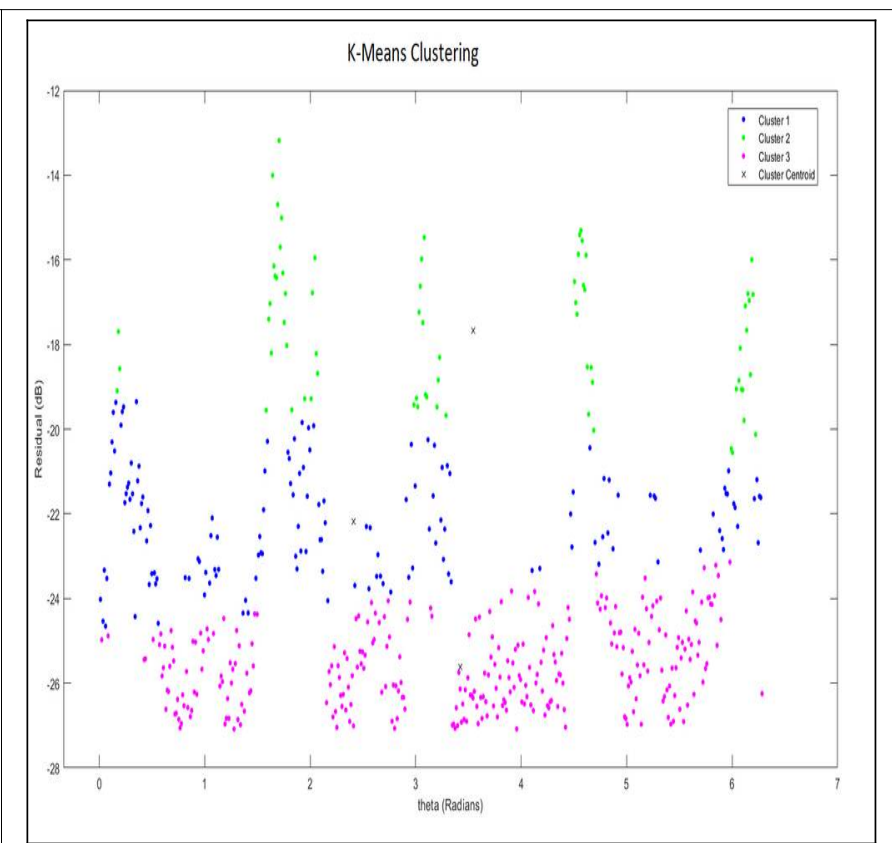

(b)

Fig. 10: Output of K-means clustering in: (a) iteration 1; and (b) iteration 2.

As a further stage in this iterative analysis, both the power domain and the ' $\theta$ ' domain values were given as inputs to the KMeans classification algorithm. Since, the clusters are initially randomized, different clustering results are obtained, each time the algorithm was run. The initial centroids in the K-means clustering must be defined and fixed to prevent arbitrary centroid distribution during each run, as this would otherwise lead to different clustering results [24]. One centroid is then initialized for each cluster. The initial position of the centroids was fixed by determining the ' $n$ ' equally spaced data points between the range of the data set. An example of this behavior is shown in Figure 10 (a and b), where the dataset is partitioned both in power domain (cluster 1 and cluster 2 vs cluster 3) and spatial domain (cluster 1 vs cluster 2). Contrasting this with the clusters in Figure 9, where the algorithm had in subsequent iterations partitioned the data only in power domain, the clusters observed in Figure 10 correspond to the algorithm that had inputs containing both power and spatial data. The variation in the output clusters are primarily attributed to the random initialization of centroids, as discussed in Section III.

From a quantitative analysis of the results using equation (5), the clusters shown in Figure 10(a) are the most optimal with the lowest Euclidean distance (2105.67), while the clusters in Figure 10(b) have a Euclidean distance of 2275.82, which is considered to be not the optimum clustering, although a partition in the power domain is expected from a qualitative analysis of the scatterers in the propagation channel. Hence, an iterative analysis approach had been used to address this issue.

\section{B. $\quad$ K-Medoids Clustering}

To minimize the effect of outliers in the clusters, and to have the centroid within the dataset, K-Medoids clustering approach was considered. Iterative analysis was performed and the silhouette score for each value of ' $k$ ' was recorded. Silhouette plots were then generated for each dataset.

The resulting silhouette plot for iterative analysis of the experimental data using K-Medoids clustering is shown in Figure 11(a). As is evident from these plots, the optimum number of clusters cannot be determined from the silhouette plot, as the plot is wildly fluctuating. While a constant value is expected for the of optimum number of clusters, having to cluster each dataset with different such values is non-ideal, considering the fact that the experiment was conducted in the same laboratory setup, i.e., constant number of scatterers in the propagation channel that are spatially invariant to the position of the transmitter antenna. This trend has also been distinctly observed for the various other off-boresight angles varying from $0^{\circ}$ to $60^{\circ}$, obtained at $15^{\circ}$ intervals. The first peak silhouette score was found with $\mathrm{k}=3$ for $\theta=15^{\circ}, 60^{\circ}$ and $0^{\circ}$. However, taking $\mathrm{k}=3$ maps the interpretation onto the earlier assumption of only 3 type of scatterers in the propagation channel, which is factually inappropriate. 
All the same, the first peaks in the other datasets, viz. of $\theta=30^{\circ}$ and $45^{\circ}$, are at $\mathrm{k}=6$.

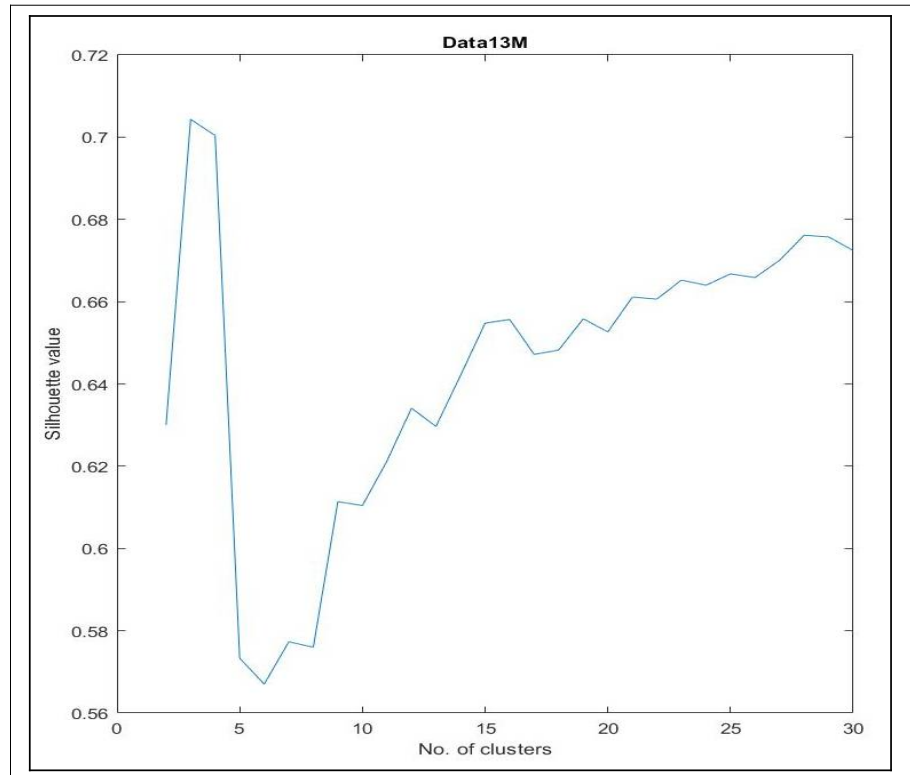

(a)

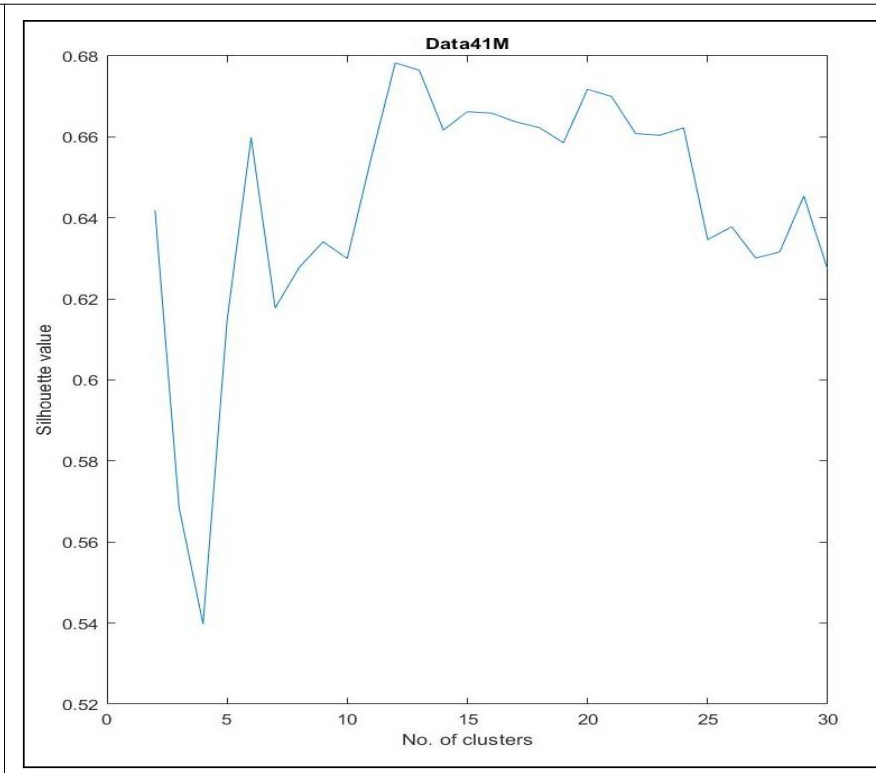

(b)

Fig. 11: Variation in the no. of clusters vs Silhouette values for: (a) $\theta=15^{\circ}$; and (b) $\theta=45^{\circ}$.

This value is appropriate for the optimum number of clusters, as it also agrees with the presence of 6 possibly distinct types of scatterers in the propagation channel. However, this value of ' $k$ ' cannot remain invariant for all the datasets since there had been a significant dip in the respective silhouette value, that clearly indicates a limitation in the clustering approach. Therefore, a definite optimum number of clusters cannot be determined from the Silhouette analysis, as that there had been a total lack of any discernible trend with the increasing number of clusters. Hence, an alternative methodology, viz. the elbow analysis, had to be employed for determining the optimum number of clusters.

\section{Elbow Method}

The Elbow method involved a computation of the WCSS, as defined by equation (7), for each value of ' $k$ '. A plot of number of clusters vs WCSS is then generated for each off-boresight dataset.

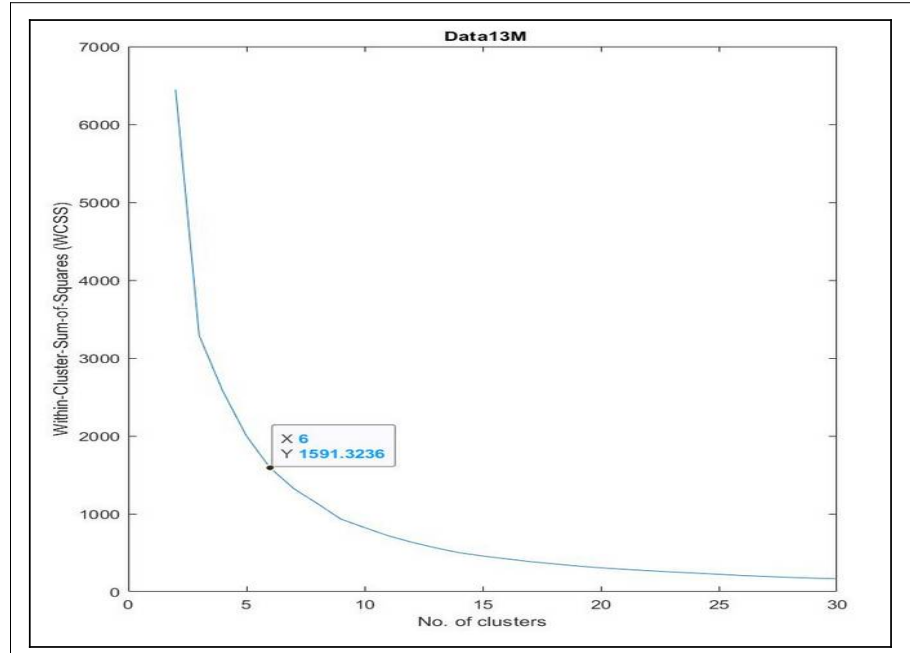

(a)

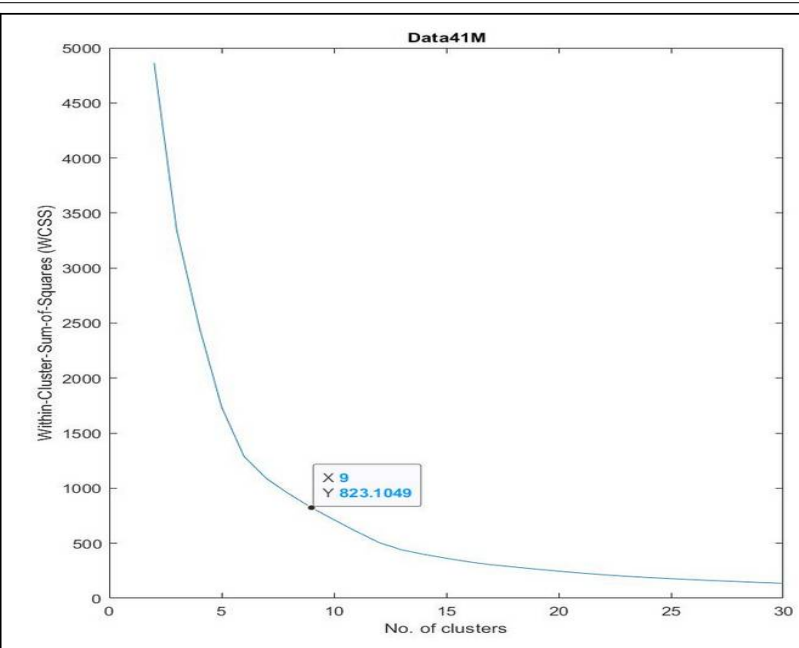

(b)

Fig. 12: Variation in the no. of clusters vs Silhouette values and (b) WCSS, for $\theta=$ (a) $15^{\circ}$ and (b) $45^{\circ}$. 
A precise selection of the point of the elbow is crucial here, since it would be an indication of the location beyond which there shall be no further significant improvement to the WCSS, i.e. the gradient of the plot starts to flatten out beyond such a point, and performing the clustering in the dataset beyond this point that is indicative of the optimal number of clusters shall lead to an increase in the computational complexity with no significant outcome. It has thus become possible to derive the optimum number of clusters from the elbow method derived plots.

Using the elbow analysis the optimum number of clusters had been determined, as depicted in the plots shown in Figure 12. It is observed that the elbow is set between 6 and 9 for all datasets - the set of points of the elbows is $\{6,7,7,8,9\}$. Although the elbow method uses quantitative formulations to explain the variation in WCSS with the number of clusters, an inference on the point of the 'elbow' is highly subjective. From similar such elbow plots for the various other off-boresight angles varying from $0^{\circ}$ to $60^{\circ}$, obtained at $15^{\circ}$ intervals, the optimum number of clusters was found to be 7 .

It has been observed from all similar such plots that the choosing of a median value of 7 for further analysis does not affect clustering as there is not much change in WCSS. Moreover, since the interpretation of the elbow is qualitative, a value of 7 for the optimal number of clusters is acceptable. Hence, for all further analysis, a median value of 7 had been chosen. All the same, in order to accommodate a potential scale-up in the number of power levels, considering a fairly complex propagation scenario, the number of clusters is enhanced by a factor of about 2 - to a value of 15 . This also enables a concomitant increase in the detection accuracy for

several diverse scatterers in the propagation channel, each leaving a unique signature defined as part of its interaction with the RF signal that enables an enhanced estimation of its scattering characteristics.

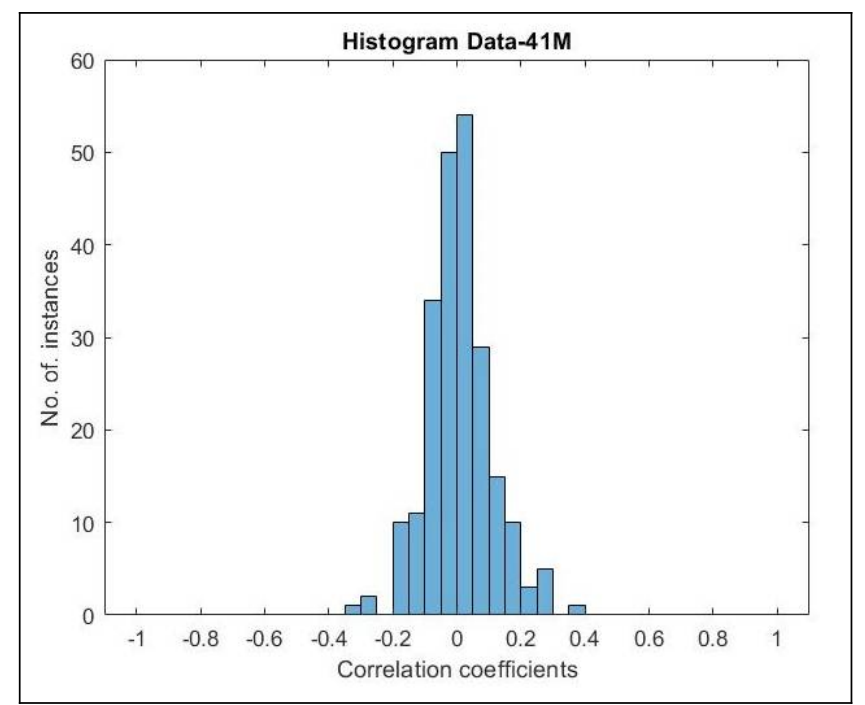

Fig. 13: Histogram of correlation coefficients $\theta=45^{\circ}$.

From the elbow plots it is observed that an increase in the optimal number of clusters to 15 causes a decrease in the value of WCSS by $50 \%$. This means that the individual clusters have a smaller footprint in the clustering plot when compared to clusters that were partitioned using $\mathrm{k}=7$. It is necessary to increase the resolution of detection due to the high degree of variability in the scatterers located within of the laboratory. A

good example of why increasing the resolution of clustering is beneficial in the analysis is the enhanced ability offered during the detection of a wider spectrum of spatial scatterers that impact the signal propagation even for the case of a single offboresight dataset.

\section{Correlation Coefficients}

The clustering was done using $\mathrm{k}=15$ and the correlation coefficients between such clusters were determined as discussed in Section III-G. In order to study the distribution in the correlation coefficient values, the related histograms were generated. The bin size was set as 0.05 , and correlation coefficient values that fall within that window are aggregated to create the histogram plots. The resulting histograms, for the case of off-boresight angles varying from $0^{\circ}$ to $60^{\circ}$, obtained at $15^{\circ}$ intervals, are similar to the one shown in Figure 13 for the specific case of $\theta=45^{\circ}$. 
TABLE II

STATISTICAL PARAMETERS IN THE HISTOGRAMS

\begin{tabular}{cccc}
\hline \hline Angular dataset & Mean & Median & $\begin{array}{c}\text { Standard } \\
\text { Deviation }\end{array}$ \\
\hline$\theta=15^{\circ}$ & 0.0026 & 0.0049 & 0.1180 \\
$\theta=30^{\circ}$ & 0.0091 & -0.0089 & 0.1052 \\
$\theta=45^{\circ}$ & 0.0073 & 0.0017 & 0.1025 \\
$\theta=60^{\circ}$ & 0.00097 & -0.00053 & 0.1083 \\
& & & \\
\hline \hline
\end{tabular}

The statistical parameters derived from the histogram distributions depicted in all plots similar to the one in Fig. 13 are listed in Table II. It is observed from the various histograms, and from a consolidation of the various statistical

moments in Table II, that the number of instances where the clusters show no correlation is significantly higher. The inference on such an outcome is based on the fact that the correlation coefficient between the clusters shall be a high value only when clusters with similar points are compared, which in this case is very unlikely given the random nature of K-Medoids clustering. In all of the other spatially distributed datasets, the correlation coefficients shall be very nearly 0 . This shall corroborate the fact that any noise component is primarily uncorrelated at the Rx. One limitation of the correlation coefficient distribution study is that it is limited to the detection of linear relationships between a pair of datasets. Hence, it is incapable of detecting any non-linear relationships. The absence of correlation coefficients values beyond a \pm 0.4 indicates that no clusters from the angular dataset precisely match those of the boresight dataset, and there are no outliers in these datasets.

\section{E. Bar chart}

Data points belonging to similar clusters were grouped together across all five data-sets and bar charts were then plotted to visualize the spatial distribution of the cluster and their frequency of occurrence. The peaks in the bar charts of Figure 14 indicate the role of known scatterers at the specific spatial locations.

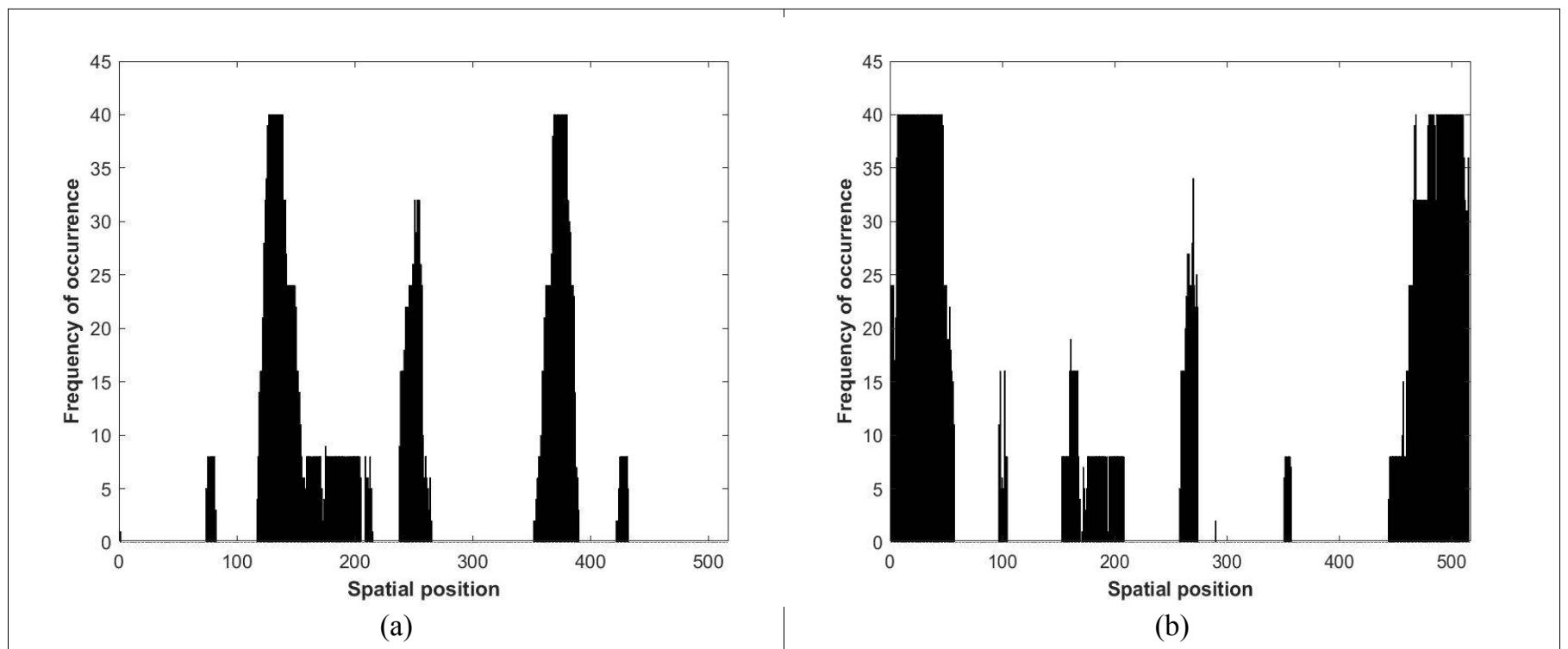

Fig. 14. Spatial occurrences of data points belonging to: (a) cluster red, representing scatterers with absorbing property; and (b) cluster green, representing scatterers with reflecting property.

The results have been a vital indication of the dimensions and locations of prominent scatterers in the laboratory environment, as had been depicted in Figure 1. It is also observed from these plots that there are spatial distributions associated with signals of uncatalogued types, inferred from the presence of smaller peaks. These could be reliable indications of unclassified "targets", and these occur due to the presence of human interference in the operating environment, the effect due to various other electronic devices, or the movement made to a few other small objects such as chairs and tables during the course of the experiment within the laboratory confines.

The spatial distributions of the absorb and reflect type scatterers in the test environment, that has resulted in effects such as signal fading and signal enhancement, respectively, were identified from the bar charts in Figure 14 (a and b). The spatial distributions are then transformed into the respective angular co-ordinates, considering the relevance of the spherical reference 
frame to the signal propagation analysis exercise. This has further helped with obtaining the locations, and also in their correlation to the equivalent physical dimensions, of prominent scatterers - such as metallic cupboards, work benches, window grates and doors, that had been already identified in the floor plan of the laboratory as depicted in Figure 1.

\section{F. Box Plot}

For each data interval, the median spatial positions were determined, and used to generate the box plots. A representative plot for the median value of 251 in the spatial position associated with the TX:RX-1 (Figure 2) radiation pattern data is shown in Figure 15. Salient indicators regarding the scatterer morphology, location, and temporal variability of their absolute position are then inferred from the five-number summary reported in similar such box plots that were generated for a total of 5 cases.

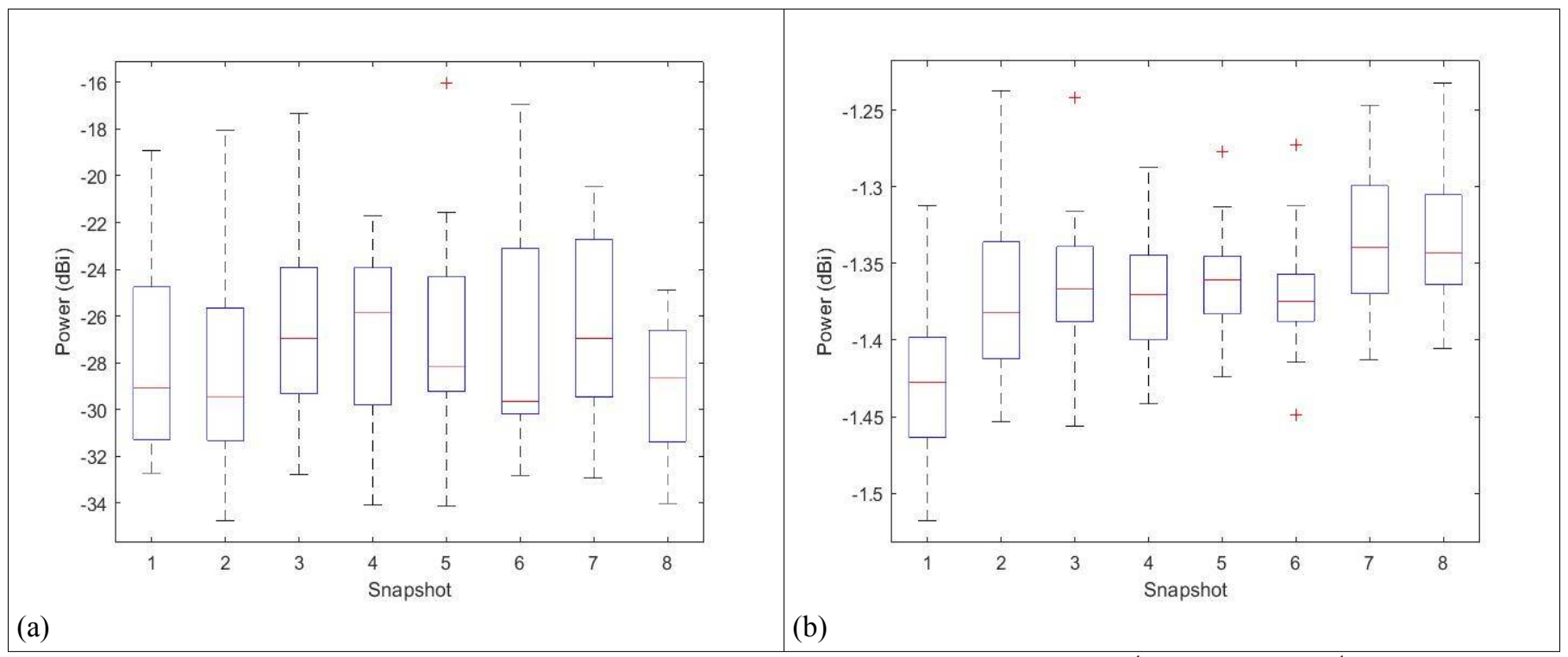

Fig. 15. Box plots of the variation of Rx power across the 8 snapshots in the data, taken at: (a) the $251^{\text {th }}$; and (b) the $210^{\text {th }}$ spatial positions.

The boxes in Figure 15(a) show a higher variation in radiated power when compared to the ones in Figure 15(b). With reference to the $210^{\text {th }}$ spatial position in the TX:RX-1 data, the box plots in all the other RX-2 positions look similar to the one shown in Figure 15(b) across all 5 experimental data sets, which is an indication that there had been a negligible effect due to the known scatterers on the propagating signal. The range of the boxes is also quite low as can be inferred from the ordinate values in this plot.

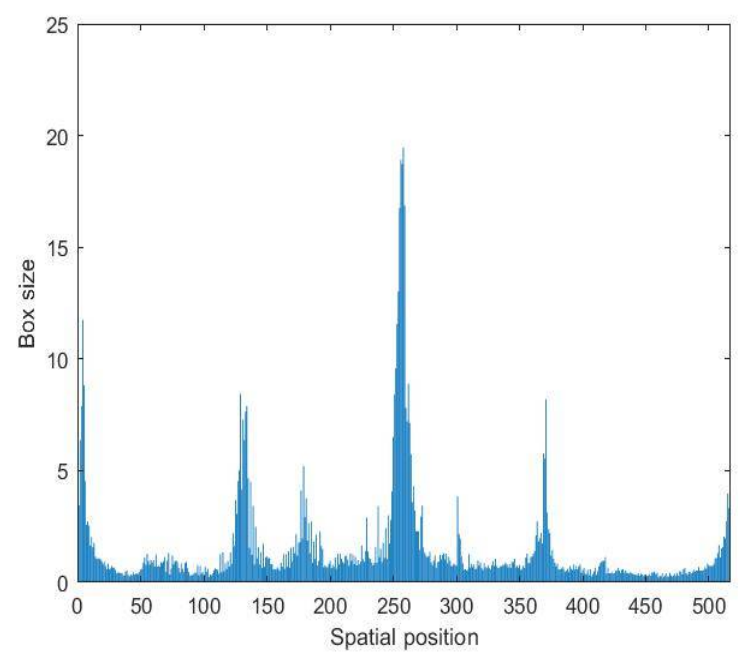

Fig. 16. Variation of box size as a function of the spatial positions.

The outcome of such analyses confirms the existence of scatterers, and their presence is evidenced from the reliable signature of the tell-tale scattering phenomenon noticed at specific angular ranges in the spatial distributions. There is further proof offered 
by the fact that the snapshots were taken at the same spatial locations, and within a very small time interval and, yet, the data sets exhibit large variations in the received power.

A further step in the analysis had been to relate the sizes of the boxes, in plots such as in Figure 15, to the non-LoS arrival paths of RF signals that correspond to the physical location of the prominent scatterers. The results from this are depicted in Figure 16. It becomes immediately obvious from the plot that any relative variation were insignificant at positions where the scatterers were absent. Similar plots were also generated for all the 5 experimental data sets that correspond to the various offboresight angles $\theta$, and the result pertaining to the scatterer profiles were found to be consistent among these plots as well, and all of them exhibited a trend that is similar to the one shown in Figure 16.

In the final analysis, using the bar charts and the box plots, the scatterers had been defined in more quantitative terms, and with enhanced certainty about their positions and characteristics. The results of such a quantitative analysis is in agreement with the outcomes of many other intensive data-driven approaches, starting with the K-means clustering scheme, that had already been invoked to characterize the scatterers by identifying and deploying a suitable ML strategy on the dataset.

\section{CONCLUSIONS}

The objective of this research work had been to profile scatterers in the propagation channel using experimental data collected in a routine laboratory environment, with the systematic application of various data-driven approaches, in a contrast to the conventional analytical model-driven schemes, in order to characterize various scatterers in the C-band signal propagating channel. The data pre-processing techniques worked in conjunction with K-means clustering to yield a good quality result. This was evident from the fact that the dimensions of the room and the known scatterers in this signal space were found to agree with the distinctive patterns they had created in the received signals, and this was inferred from the experimental results. Hence, a simultaneous qualitatively characterizing and quantitatively determining of the influence of the scattering centers on the propagating signal, based on their material attributes, morphology, and kinematics - has been inferred from an offline processing of the tracked time series data. In situations that involve intentional incorporation of technologies that help to evade detection of aerospace targets of interest in a given surveillance space, the proposed method has the significant advantage of sensing such targets from the signatures of change in the reference signal in the wake of their interaction with the propagation channel.

As a possible extension of the work reported here, with a precise understanding of the scatter signatures obtained from the trends and patterns in the acquired data and then fed back in real time, a test system can perform in a manner so as to constrain its acquisition parameters dynamically. With such a closed-loop scheme, the malicious components in a sensitive RF signal detection procedure can now be subjected to adaptive excision, a task that is currently being pursued at an exorbitant cost and time span in facilities such as the anechoic chamber. The profiling of antennas with unknown radiation characteristics will then become a wholly computationally defined task, that shall prove definitively cost effective as the process gets refined during the testing phases, in terms of noise detection, with its progressive exposure to further data sets as part of supervised learning and classification exercises.

Information sharing being the fundamental tenet in network-centric warfare (NCW), a combination of technologies that encompass multistatic RaDaR sensors, communication signals and systems, data processing, and data management shall play a pivotal role in establishing resilient links among assets that are aerospace and ground based. The evident benefits of such a robust detection and communications backbone in strategic and tactical aerospace applications is in the form of timely, effective and safe operations over the course of an entire mission. Ad-hoc and highly dynamic base station densification operations in the context of NCW have to be initiated in an extensive manner with the aim of supporting complex radio techniques in $5 \mathrm{G}$ that need to offer enhanced data rates, capacity and coverage in the macro and micro spatial scales. Incorporation of ML technique and strategies discussed in this work, in concert with high quality situational awareness models, shall then yield rapid and time-bound estimates on theater-specific target attributes at the locations of aerospace-based antenna terminals during the mid-band 5G-NR NCW operations. These shall in effect serve as potent replacement solution to the various conventional methods that require extensive modeling and outdoor test range studies at elevated costs and human effort.

\section{REFERENCES}

1 W. Wang, G. Hoerack, T. Jost, R. Raulefs, M. Walter and U. Fiebig, "Propagation channel at $5.2 \mathrm{GHz}$ in Baltic Sea with focus on scattering phenomena," in 2015 9th European Conf. Antennas Propagat. (EuCAP), Lisbon, 2015, pp. 1-5.

2 Y. Karasawa, M. Yamada and J. E. Allnutt, "A new prediction method for tropospheric scintillation on Earth-space paths," IEEE Trans. Antennas Propagat., vol. 36, no. 11, pp. 1608-1614, 1988.

3 S. Srivatsa and G. A. Shanmugha Sundaram, "PAM4-Based RADAR Counter-Measures in Hostile Environments"; In: Thampi S M et al. (Eds.) Proceedings of 3rd ISTA 2017; Advances in Intelligent Systems and Computing 683(1): Intelligent Systems Technologies and Applications, Springer International Publishing AG, pp. 390-400, 2018.

4 P. A. van Walree, "Propagation and Scattering Effects in Underwater Acoustic Communication Channels," IEEE J. Oceanic Eng., vol. 38, no. 4, pp. 614631, 2013. 
5 J. Hao, Z. Jie and K. Hisakazu, "Generalised three-dimensional scattering channel model and its effects on compact multiple-input and multiple-output antenna receiving systems", IET Comm., vol. 9, pp. 2177-2187, 2015.

6 J. S. Lu, X. Han and H. L. Bertoni, "The Influence of Terrain Scattering on Radio Links in Hilly/Mountainous Regions," IEEE Trans. Antennas Propagat., vol. 61 , no. 3, pp. 1385-1395, 2013.

7 W. Y. Lee, "Effects on correlation between two mobile radio base-station antennas," IEEE Trans. Vehic. Technol., vol. 22, no. 11, pp. 1214-1224, 1973.

8 A. Maric, E. Kaljic and M. Hadzialic, "A Unified Hollow-Disk Scattering Model: Angle-of-Departure and Time-of-Arrival Statistics," IEEE Trans. Vehic. Technol., vol. 68, no. 5, pp. 4342-4352, 2019.

9 L. Cheng, D. D. Stancil and F. Bai, "A Roadside Scattering Model for the Vehicle-to-Vehicle Communication Channel," IEEE J. Sel. Areas Comm., vol. 31, no. 9, pp. 449-459, 2013.

10 S. Sundaresan and G. A. Shanmugha Sundaram, "Simulation Study on Modeling the Effects of Wind Turbine on Communication Signals (C and X bands) using XGtd", Proceedings of ICCSP 2015, IEEE, pp. 173-77, 2015.

11 VIAVI Solutions Inc., Application Note: The Role of C-band in 5G [Online]. Available: https://www.viavisolutions.com/en-us/literature/role-c-band-5gapplication-notes-en.pdf [Accessed: 10-Apr-2021].

12 Ettus Research, a National Instruments Brand: The leader in Software Defined Radio (SDR), VERT2450 Antenna [Online]. Available: https://www.ettus.com/all-products/vert2450 [Accessed: 14-Mar-2019].

13 National Instruments, USRP-2954 [Online]. Available: https://www.ni.com/en-in/support/model.usrp-2954.html [Accessed: 14-Mar-2019].

14 National Instruments, PXIe-1082 [Online]. Available: https://www.ni.com/en-in/support/model.pxie-1082.html [Accessed: 14-Mar-2019].

15 National Instruments, NI LabVIEW [Online]. Available: https://www.ni.com/en-us/shop/labview/select-edition.html [Accessed: 14-Mar-2019].

16 National Instruments, LabVIEW Communications System Design Suite [Online]. Available: https://www.ni.com/en-us/shop/select/labviewcommunications-system-design-suite [Accessed: 14-Mar-2019].

17 Shield_Motor-REV3c [Online]. Available: https://www.arduino.cc/en/uploads/Main/arduino_MotorShield_Rev3-schematic.pdf [Accessed: 14-Mar-2019].

18 Raspberry Pi 2 Model B [Online]. Available: https://www.raspberrypi.org/products/raspberry-pi-2-model-b [Accessed: 01-Jul-2019].

19 R. Harjani and T. A. Lee, "FRC: A method for extending the resolution of Nyquist rate converters using oversampling," IEEE Trans. Circuits Syst. II Analog Digit. Signal Process., vol. 45, no. 4, pp. 482-494, 1998.

20 S. A. Hanna, "Convolutional interleaving for digital radio communications," in Proceedings of 2nd IEEE International Conference on Universal Personal Communications, 1993, vol. 1, pp. 443-447.

21 S. Rapuano and F. J. Harris, "An introduction to FFT and time domain windows," IEEE Instrum. Meas. Mag., vol. 10, no. 6, pp. $32-44,2007$.

22 Datastore," Datastore - MATLAB \& Simulink. [Online]. Available: https://www.mathworks.com/help/matlab/datastore.html. [Accessed: 01-Jul-2019].

23 R. Jolly and G. A. Shanmugha Sundaram, "Phased antenna array design for L-band aerospace detection of radiolytic components", Proceedings of 11th International Conference on Signal Processing and Communications (SPCOM) 2016, IEEE Inc., pp. 81-5, 2016.

24 C. A. Balanis, Antenna Theory Analysis and Design, 3rd ed. Wiley-Interscience: New York, 2005, ch. 2.

25 T. Velmurugan and T. Santhanam, "Computational Complexity between K-Means and K-Medoids Clustering Algorithms for Normal and Uniform Distributions of Data Points," Journal of Computer Science, vol. 6, pp. 363-368, 2010.

26 K. L. Du, "Clustering: A neural network approach," Neural Networks, vol. 23, no. 1, pp. 89-107, 2010

27 T. Prvan, "Essentials of Multivariate Data Analysis. By N. H. Spencer Boca Baton, Florida CRC Press 2014186 pages. UK £35.99 (paperback). ISBN $978-$ 1-4665-8478-5," Australian \& New Zealand Journal of Statistics, vol. 57, no. 4, pp. 574-575, 2015.

28 P. J. Rousseeuw, "Silhouettes: A graphical aid to the interpretation and validation of cluster analysis," J. Comput. Appl. Math., vol. 20, no. C, pp. 53-65, Nov. 1987.

29 L. Kaufman and P. J. Rousseeuw, Finding Groups in Data: An Introduction to Cluster Analysis, Hoboken, NJ, USA: Wiley, 2009.

30 H. W. Choi, N. M. F. Qureshi, and D. R. Shin, "Comparative Analysis of Electricity Consumption at Home through a Silhouette-score prospective," in 2019 21st International Conference on Advanced Communication Technology (ICACT), 17-20 Feb. 2019 2019, pp. 589-591.

31 Z. Liu, Q. Pan, J. Dezert, J. Han and Y. He, "Classifier Fusion With Contextual Reliability Evaluation," IEEE Trans. Cybernet., vol. 48, no. 5, pp. 1605$1618,2018$.

32 C. L. Clayman, S. M. Srinivasan and R. S. Sangwan, "K-means Clustering and Principal Components Analysis of Microarray Data of L1000 Landmark Genes," Procedia Computer Science, vol. 168, pp. 97-104, 2020/01/01/2020.

33 N. Arbin, N. S. Suhaimi, N. Z. Mokhtar and Z. Othman, "Comparative Analysis between K-Means and K-Medoids for Statistical Clustering," in 2015 3rd International Conference on Artificial Intelligence, Modelling and Simulation (AIMS), 2-4 Dec. 2015 2015, pp. 117-121.

34 B. Ratner, "The correlation coefficient: Its values range between $+1 /-1$, or do they?," J. Targeting, Meas. Anal. Market. vol. 17, no. 2, pp. 139-142, 2009/06/01 2009.

35 Five-Number Summary and Box-and-Whisker Plots [Online]. Available: http://web.mnstate.edu/peil/MDEV102/U4/S36/S36_print.html [Accessed: 22Jan-2019]. 